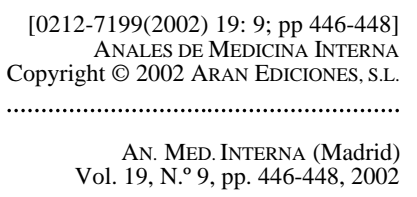

\section{Adecuación de los ingresos hospitalarios urgentes}

\author{
J. OCHOA-GÓMEZ, A. VILLAR ARIAS, E. RAMALLE-GÓMARA*, \\ J. M. CARPINTERO ESCUDERO, L. BRAGADO BLAS, J. I. RUIZ AZPIAZU \\ Servicio de Urgencias del Hospital San Millán de Logroño. *Servicio de Epidemiología y \\ Promoción de la Salud del Gobierno de La Rioja. Logroño. La Rioja
}

\begin{abstract}
RESUMEN
Objetivo: Estudio realizado para conocer el porcentaje de ingresos inapropiados urgentes de un hospital general.

Material y métodos: Estudio descriptivo retrospectivo de los pacientes ingresados durante 1.999 mediante aplicación del Appropriateness Evaluation Protocol (AEP) para valorar la adecuación del ingreso.

Resultados: Dieciocho pacientes $(4,5 \%)$ ingresaron de forma inapropiada. Hubo mayor riesgo de ingreso inadecuado por los especialistas médicos (internista de guardia, hematólogo o nefrólogo): odds ratio frente a ingreso por médico de Urgencias de 5,3 $(\mathrm{p}<0,03)$.

Conclusiones: Destaca una bajo porcentaje de ingresos inadecuados $(4,5 \%)$. Existe mayor riesgo cuando el médico responsable del ingreso pertenece a una especialidad médica.
\end{abstract}

PALABRAS CLAVE: Ingreso inadecuado. Urgencias. Especialidad médica.

\author{
APPROPRIATENESS OF THE EMERGENT HOSPITALARY \\ ADMISSIONS
}

\begin{abstract}
Introduction: The aim of this work is to know the proportion of ina dequate urgent admissions in a general hospital.

Material and methods: A retrospective and descriptive study of patients admitted during 1,999 was performed. The Appropriatness Eva luation Protocol (AEP) was used in order to evaluate the appropriatness of the admissions.

Results: Eighteen admissions (4.5\%) were inadecuated. There was a higher proportion of inappropriated admission (IA) when the physician responsible of the admission was a medical specialist (internal medicine, hematology or nephrology): odds ratio 5.3 in opposite to emergency phy sicians ( $p<0.03)$.

Conclusions: There was a low proportion of inadequate admissions (4.5\%) and the risk of inappropriatness of the admission was major when it was ordered by a medical specialist.
\end{abstract}

KEY WORDS: Inadecuated admission. Emergency room. Medical spe ciality.

Ochoa-Gómez J, Villar Arias A, Ramalle-Gómara E, Carpintero Escudero JM, Bragado Blas L, Ruiz Azpiazu JI. Adecuación de los ingresos hospitalarios urgentes. An Med Interna (Madrid) 2002; 19: 446-448.

\section{INTRODUCCIÓN}

Los Servicios de Urgencias (SU) de los hospitales son cada vez más utilizados por la población española. En 1998, los SU dependientes del INSALUD atendieron a más de 5 millones de personas (1).

Entre los objetivos del SU se encuentra determinar qué pacientes requieren ingreso hospitalario para su diagnóstico y tratamiento. Además, la búsqueda de la eficiencia obliga a vigilar el empleo inapropiado del ingreso hospitalario como recurso sanitario. El Appropriateness Evaluation Protocol (AEP) (2) es un cuestionario formado por 18 items que se ha mostrado como una herramienta de gran fiabilidad (3) para cuantificar los ingresos hospitalarios innecesarios, aunque tiende a sobrevalorar -respecto al juicio de los clínicos- el por- centaje de dichos ingresos. EL AEP ha sido validado en España $(4,5)$ y tiene una buena sensibilidad, especificidad y reproductibilidad interobservador (índice Kappa $=0,48-0,59$ ) e intraobservador $(0,66-0,88)$.

El objetivo de este trabajo es conocer, mediante la aplicación del AEP, el porcentaje de ingresos inapropiados urgentes de un hospital general y si existe asociación entre el tipo de médico responsable del ingreso y la utilización inadecuada de los ingresos urgentes.

\section{PACIENTES Y MÉTODOS}

Estudio descriptivo retrospectivo realizado sobre una muestra representativa, seleccionada mediante una tabla de

Trabajo aceptado: 14 de mayo de 2002

Correspondencia: F. Javier Ochoa Gómez. Servicio de Urgencias. Hospital San Millán. C/Autonomía 3. 26004 Logroño (La Rioja). Tfno.: 941 294 500 (Ext. 527 y 528). e-mail: jochoa@nacom.es 
TABLA I

INGRESOS INAPRO PIADOS, SEGÚN EL M ÉDICO RESPO NSABLE DEL INGRESO

\begin{tabular}{|c|c|c|c|c|}
\hline & \multicolumn{2}{|c|}{ Ingreso apropiado } & \multicolumn{2}{|c|}{ Ingreso inapropiado } \\
\hline & $n$ & $\%$ & $n$ & $\%$ \\
\hline M édico adjunto de Urgencias & 194 & 95,1 & 10 & 4,9 \\
\hline $\begin{array}{l}\text { Especialista "quirúrgico": } \\
\text { cirujano, ORL, oftalmólogo, } \\
\text { urólogo, ginecólogo } \\
\text { traumatólogo }\end{array}$ & 129 & 97,7 & 3 & 2,3 \\
\hline M edicina intensiva & 14 & 100 & 0 & 0 \\
\hline Pediatra & 30 & 93,8 & 2 & 6,2 \\
\hline $\begin{array}{l}\text { Especialista " médico" : internista } \\
\text { de guardia, hematólogo, } \\
\text { nefrólogo }\end{array}$ & 13 & 81,3 & 3 & 18,7 \\
\hline
\end{tabular}

Chi ${ }^{2}$ de Pearson: 10,4 grados de libertad, $p=0,04$

números aleatorios, de los pacientes ingresados durante 1999 en un hospital de segundo nivel. Para el cálculo del tamaño muestral se asumió una prevalencia de ingresos inapropiados del $17 \%$ (6), un nivel de confianza del $95 \%$ y una precisión del 3,5\%. Se estableció como representativa una muestra de 412 pacientes ingresados por el SU, una vez incorporada una corrección para poblaciones finitas.

El hospital tiene 550 camas y sirve de referencia a una Comunidad Autónoma uniprovincial de 265.000 habitantes. Su Servicio de Urgencias atendió en 1999 a 85.000 personas, de las que casi un 14\% requirió ingreso hospitalario.

La plantilla del SU está formada por 15 médicos adjuntos: 13 son especialistas en Medicina Familiar y Comunitaria y 2 en Medicina Interna. Los ingresos desde el SU son decididos por ellos, a excepción de los de pacientes del Area Quirúrgica, Pediatría, Hematología, Medicina Intensiva y Nefrología. En algunos casos, se consulta a especialistas de Medicina Interna acerca de la conveniencia de un determinado ingreso, o ellos envían pacientes para ingresar desde consultas, y son ellos, en estos casos, quienes lo indican.

En cada paciente se estudiaron: edad, sexo, domicilio (ciudad en la que está ubicado el hospital, otras localidades de la provincia o fuera de la provincia), hora de ingreso (agrupadas en turno de mañana, tarde o noche), día de la semana (de lunes a domingo), mes y especialidad del médico (urgencias, pediatra, cuidados intensivos, especialista médico - nefrólogo, internista o hematólogo - y especialista quirúrgico - cirujano, ORL, oftalmólogo, urólogo, ginecólogo, traumatólogo) que ordenó el ingreso.

A todos los pacientes se les aplicó el cuestionario AEP considerando que el ingreso era inapropiado cuando no cumplía alguno de los criterios. En el caso de menores de 14 años se les aplicó la versión pediátrica del protocolo "Pediatric Appropriatness Evaluation Protocol" (PAEP) validada por otros autores (7). Para la estadística descriptiva se emplearon la media y desviación estándar o la proporción, en función del tipo de variable (cuantitativa o cualitativa). La fuerza de asociación entre la variable resultado (ingreso apropiado o no) y cada una de las variables consideradas se evaluó con la odds ratio (OR), mediante un análisis de regresión logística.

\section{RESULTADOS}

Se consiguió acceder a 398 de las 412 historias seleccionadas de forma aleatoria $(96,35 \%)$. La media de edad de los pacientes fue de 58,6 años ( $\mathrm{DE}=25,2)$ con un rango entre 0 y 97 años. Ciento setenta y dos pacientes $(43,2 \%)$ fueron mujeres.

La distribución de los pacientes hospitalizados en función del médico que ordenó su ingreso se presenta en la tabla I.

Dieciocho pacientes $(4,5 \%)$ ingresaron de forma inapropiada, pues no cumplían ningún criterio del cuestionario AEP. La única variable asociada con mayor probabilidad estadística de ingreso inadecuado fue que el ingreso estuviera indicado por un facultativo perteneciente a una especialidad médica (internista de guardia, hematólogo o nefrólogo): odds ratio frente a ingreso por médico adjunto de Urgencias de 5,3 ( $\mathrm{p}<0,03$ ).

Los principales motivos de ingreso inapropiado fueron: posibilidad de realizar los procedimientos diagnósticos o terapéuticos sin necesidad de ingreso hospitalario (50\%), imposibilidad de programar la prueba sin ingreso (aunque sea posible realizarla de forma ambulatoria) $(22,5 \%)$, necesitar cuidados de menor nivel asistencial que los de un hospital de agudos $(22,5 \%)$ y vivir lejos del hospital (5\%).

\section{DISCUSIÓN}

En nuestro estudio destaca una bajo porcentaje de ingresos inadecuados $(4,5 \%)$ - evaluados mediante la aplicación del AEP - comparado con otros hospitales de nuestro país: 17,1\% de Matorras et al6 o 13,4\% de Rodríguez-Vera et al (8).

Como en estudios previos $(8,9)$ no hubo diferencias en los ingresos incorrectos atribuibles a la edad de los pacientes, aunque en otros trabajos realizados en España se ha determinado un mayor riesgo de ingreso inapropiado en los pacientes más jóvenes (4). Tampoco la hora, día o mes de ingreso se asociaron en nuestro estudio a mayor probabilidad de ingreso inadecuado.

El único factor que se asoció a una mayor probabilidad de ingreso inadecuado fue la especialidad del médico que ordenaba el ingreso; así, cuando el ingreso lo indicó un especialis- 
ta "médico" hubo un mayor "riesgo" de ingreso inadecuado. Este aspecto, no valorado en otros trabajos, puede estar relacionado con el bajo porcentaje de ingresos inapropiados de nuestro estudio. Cuando se analizan por separado los pacientes ingresados en nuestra serie por especialistas "médicos", la proporción de ingresos inapropiados es similar a la de otras series españolas (publicadas por médicos de Servicios de Medicina Interna) (6).

Las limitaciones del trabajo son las propias del AEP (10) y las derivadas del tamaño muestral, que determinó una casuística limitada: dieciocho ingresos inadecuados.

La mayor proporción de ingresos inadecuados entre los facultativos de especialidades médicas, respecto a otros (intensivista, médico de Urgencias, especialistas "quirúrgicos") puede deberse a su mayor tendencia a ingresar a los pacientes para "estudio", dada la lentitud de las exploraciones complementarias por vía ambulatoria. Una parte de estos ingresos inapropiados podría evitarse mediante la canalización de la demanda hacia otros niveles asistenciales (dotando a la Atención Primaria de un mayor - y más rápido - acceso a pruebas complementarias) o la creación de consultas hospitalarias de alta resolución (10). Es posible que además, con estas medidas se evitase sobrecargar los SU.

El estudio sugiere que los SU dotados de plantillas propias podrían ser más eficientes que aquellos que dependen de especialistas de guardia para la toma de decisiones, particularmente en pacientes con patología médica.

Los autores proponen que, además de los factores ya conocidos asociados con ingresos urgentes inapropiados (11) (edad, diagnóstico -síndrome constitucional- y procedencia del enfermo de consultas), se indague en futuros estudios el tipo de médico que propone el ingreso.

\section{Bibliografía}

1. INSALUD. Memoria de 1999; 75.

2. Gertmann PM, Restuccia JD. The Appropriateness Evaluation Protocol. A technique for assessing unnecessary days of hospital care. Med Care 1981; 19: 855-70.

3. Strumwasser I, Paranjpe NV, Ronis DL, Share D, Shell LJ. Reliability and Validity of Utilization Review Criteria. Appropriateness Evaluation Protocol. Standardized Medreview Instrument and Intensy-SeverityDischarge Criteria. Med Care 1990; 28: 95-111.

4. González-Montalvo JI, Baztán JJ, Rodríguez-Mañas L, et al. Ingreso hospitalario urgente en servicios médicos: causas, justificación, factores sociales y sus diferencias en función de la edad. Med Clin (Barc) 1994; 103: 441-4.

5. Peiró S, Meneu R, Roselló ML, Portella E, Carbonell-Sanchis R, Fernández $\mathrm{C}$, et al. Validez del protocolo de evaluación del uso inapropiado de la hospitalización. Med Clin (Barc) 1996; 107: 124-9.

6. Matorras Galán P, De Pablo Casas M, Otero García L, Alonso López F, Daroca Pérez R, Díaz-Caneja Rodríguez N. Adecuación de los ingresos en un servicio de medicina interna de un hospital de tercer nivel. Med

Clin (Barc) 1990; 94: 290-3.

7. Casanova Matutano C, Gascón Romero P, Calvo Rigual F, et al. Uso inapropiado de la hospitalización en Pediatría. Validación de la versión española del Pediatric Appropriateness Evaluation Protocol. Gac Sanit 1999; 13: 303-11.

8. Rodríguez Vera FJ, Alcoucer Díaz MR, Rodríguez Gómez FJ, Camacho Martínez T, Colchero Fernández J, Pujol De la Llave E. Adecuación de los ingresos en un servicio de Medicina Interna de un hospital de segundo nivel. An Med Interna (Madrid) 1999; 16: 277-80.

9. Mirón Canelo JA, Orozco Pedemonte JA, Alonso Sardón M, Sáenz González MC. Estimación de ingresos y estancias inadecuadas en un hospital comarcal. Rev Clin Esp 2000; 200: 654-8.

10. Ollero Baturone M. Adecuación y utilidad del ingreso hospitalario. Med Clín (Barc) 2001; 116: 655-7.

11. Zambrana García JL, Delgado Fernández M, Cruz Caparrós G, Díez García F, Martín Escalante MD, Salas Coronas J. Factores asociados a ingresos inadecuados en un servicio de medicina interna. Med Clín (Barc) 2001; 116: 652-4. 